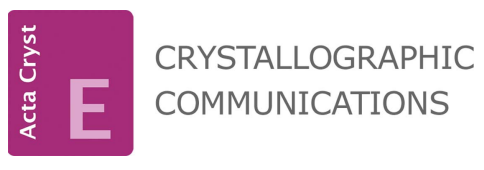

ISSN 2056-9890

Received 16 May 2017

Accepted 20 June 2017

Edited by J. T. Mague, Tulane University, USA

Keywords: crystal structure; carbazole; oxadiazolethione; hydrogen bonds; $\mathrm{C}-\mathrm{H} \cdots \pi$ (ring) contacts; $\pi-\pi$ stacking.

CCDC reference: 1557216

Supporting information: this article has supporting information at journals.iucr.org/e

\section{Crystal structure of 5-[2-(9H-carbazol-9-yl)ethyl]- 1,3,4-oxadiazole-2(3H)-thione}

\author{
Jim Simpson, ${ }^{a}$ Shaaban K. Mohamed, ${ }^{\text {b,c }}$ Talaat I. El-Emary ${ }^{\text {d }}$ and Mustafa R. \\ Albayati $^{\mathbf{e} *}$ \\ ${ }^{\mathbf{a}}$ Department of Chemistry, University of Otago, PO Box 56, Dunedin, New Zealand, ${ }^{\mathbf{b}}$ Chemistry and Environmental \\ Division, Manchester Metropolitan University, Manchester M1 5GD, England, ' ${ }^{\mathbf{C}}$ Chemistry Department, Faculty of \\ Science, Minia University, 61519 El-Minia, Egypt, 'Department of Chemistry, Faculty of Science, Assiut University, \\ 71515 Assiut, Egypt, and ${ }^{\mathbf{e}}$ Kirkuk University, College of Education, Department of Chemistry, Kirkuk, Iraq. \\ *Correspondence e-mail: shaabankamel@yahoo.com
}

The title compound, $\mathrm{C}_{16} \mathrm{H}_{13} \mathrm{~N}_{3} \mathrm{OS}$, comprises an oxadiazolethione ring bound to the $\mathrm{N}$ atom of an almost planar carbazole ring system (r.m.s. deviation = $0.0088 \AA$ ) through an ethylene chain. The oxadiazole ring is inclined to the the carbazole ring system by $40.71(6)^{\circ}$. In the crystal, $\mathrm{N}-\mathrm{H} \cdots \mathrm{O}, \mathrm{N}-\mathrm{H} \cdots \mathrm{S}, \mathrm{C}-$ $\mathrm{H} \cdots \mathrm{N}$ and $\mathrm{C}-\mathrm{H} \cdots \mathrm{S}$ hydrogen bonds combine with $\mathrm{C}-\mathrm{H} \cdots \pi(\mathrm{ring})$ and $\pi-\pi$ contacts to stack the molecules along the $b$-axis direction.

\section{Chemical context}

Carbazole derivatives have been shown to have several industrial applications including use in optoelectronic devices (Fitilis et al., 2007; Peng et al., 2011), dye-sensitized solar cells (Li et al., 2010) and photochromic dyes (Billah et al., 2008). Moreover, fused heterocycles with carbazole scaffolds are noted for their biological activities. They are found in drugs such as tubingensin $\mathrm{A}$ and $\mathrm{B}$ and have been shown to have both antiviral and cytotoxic activities (TePaske et al., 1989). The anti-inflammatory agents caprofen and etodolaca and the antipyretic agent nincazole (Ghoneim et al., 2006) are also carbazole based. The biological activity of so many carbazolebased heterocycles encouraged us to synthesize the title compound and its molecular crystal structure is reported here.

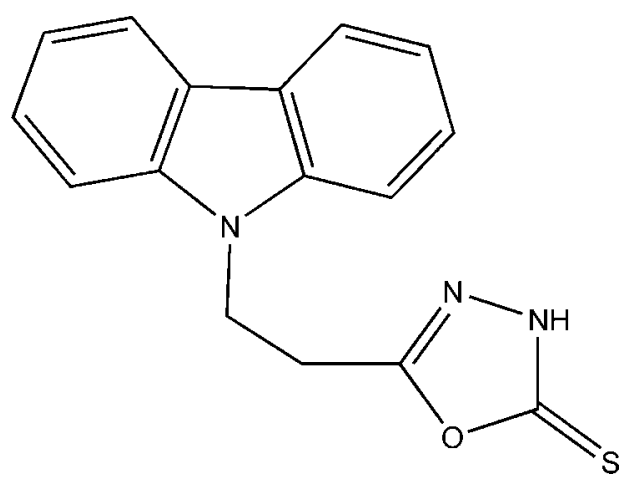

\section{Structural commentary}

In the title compound $\mathrm{C}_{16} \mathrm{H}_{13} \mathrm{~N}_{3} \mathrm{OS}$, (I), the oxadiazolethione ring binds to the carbazole ring system through a $\mathrm{C} 2-\mathrm{C} 3-\mathrm{C} 4-$ $\mathrm{N} 3$ ethylene chain with the ring systems inclined at an angle of 


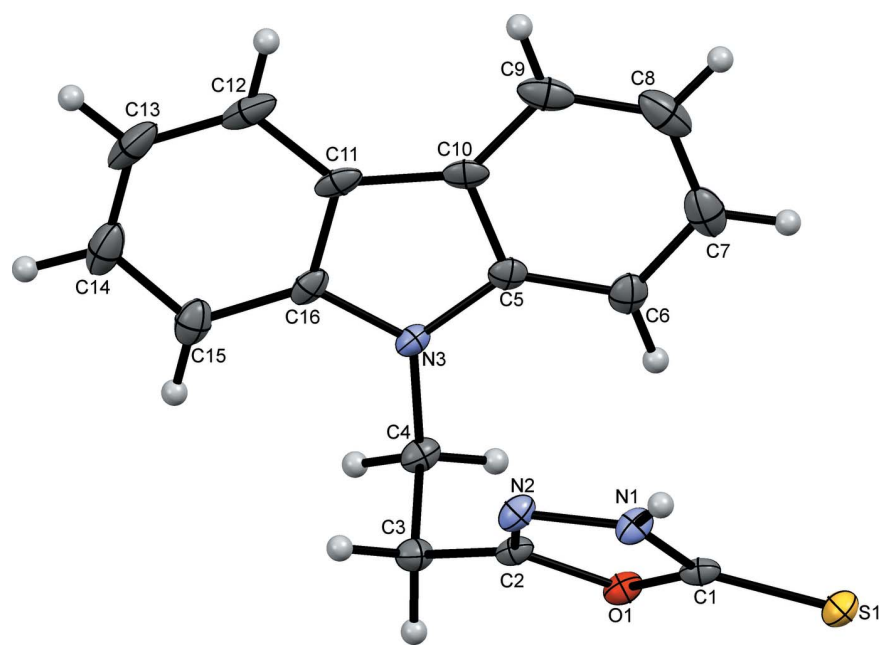

Figure 1

The molecular structure of (I) with ellipsoids drawn at the $50 \%$ probability level.

$40.71(6)^{\circ}$, Fig. 1 . The carbazole ring system is almost planar with the outer $\mathrm{C} 5-\mathrm{C} 10$ and $\mathrm{C} 11-\mathrm{C} 16$ benzene rings subtending angles of $0.38(13)$ and $0.64(13)^{\circ}$, respectively, to the central $\mathrm{N} 3 / \mathrm{C} 5 / \mathrm{C} 10 / \mathrm{C} 11 / \mathrm{C} 16$ ring. Bond lengths and angles in both ring systems are normal and similar to those found in the numerous other carbazole structures (see, for example, Kimura et al., 1985) and those of the few known oxadiazolethione derivatives with alkane chains at C5 (Khan et al. 2014; Zheng et al. 2006).

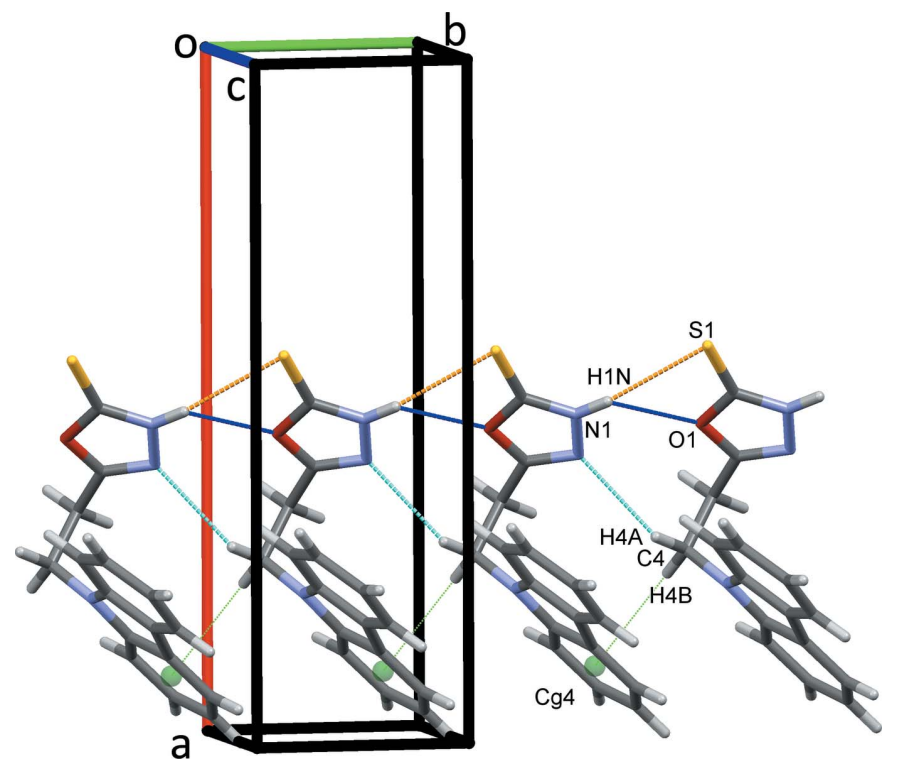

Figure 2

Rows of molecules of (I) along $b$. In this and subsequent figures, $\mathrm{N}-$ $\mathrm{H} \cdots \mathrm{S}$ (orange), $\mathrm{N}-\mathrm{H} \cdots \mathrm{O}$ (dark blue) and $\mathrm{C}-\mathrm{H} \cdots \mathrm{N}$ (light blue) hydrogen bonds are drawn as coloured dashed lines. $\mathrm{C}-\mathrm{H} \cdots \pi$ contacts are shown as green dotted lines with ring centroids displayed as coloured spheres.
Table 1

Hydrogen-bond geometry $\left(\AA,^{\circ}\right)$.

$\mathrm{Cg} 4$ is the centroid of the $\mathrm{C} 11-\mathrm{C} 16$ ring.

\begin{tabular}{lllll}
\hline$D-\mathrm{H} \cdots A$ & $D-\mathrm{H}$ & $\mathrm{H} \cdots A$ & $D \cdots A$ & $D-\mathrm{H} \cdots A$ \\
\hline $\mathrm{N} 1-\mathrm{H} 1 N \cdots \mathrm{S} 1^{\mathrm{i}}$ & $0.89(2)$ & $2.75(2)$ & $3.6053(14)$ & $162.8(19)$ \\
$\mathrm{N} 1-\mathrm{H} 1 N \cdots \mathrm{O} 1^{\mathrm{i}}$ & $0.89(2)$ & $2.62(2)$ & $3.0707(18)$ & $112.5(16)$ \\
$\mathrm{C} 3-\mathrm{H} 3 B \cdots \mathrm{S} 1^{\mathrm{ii}}$ & 0.99 & 2.93 & $3.9061(16)$ & 169 \\
$\mathrm{C} 4-\mathrm{H} 4 A \cdots \mathrm{N} 2^{\mathrm{iii}}$ & 0.99 & 2.67 & $3.495(2)$ & 141 \\
$\mathrm{C} 4-\mathrm{H} 4 B \cdots C g 4^{\mathrm{iii}}$ & 0.99 & 2.87 & $3.4577(17)$ & 119 \\
$\mathrm{C} 12-\mathrm{H} 12 \cdots C g 4^{\mathrm{i}}$ & 0.95 & 3.22 & $4.073(2)$ & 151 \\
\hline
\end{tabular}

Symmetry codes: (i) $x, y+1, z$; (ii) $-x+1,-y,-z+1$; (iii) $x, y-1, z$.

\section{Supramolecular features}

In the crystal, classical $\mathrm{N} 1-\mathrm{H} 1 N \cdots \mathrm{O} 1$ and $\mathrm{N} 1-\mathrm{H} 1 N \cdots \mathrm{S} 1$ hydrogen bonds form $C(4)$ chains of molecules linked in a head-to-head fashion along the $b$-axis direction, Fig. 2 . These contacts are bolstered by the $\mathrm{C} 4$ atom acting as a bifurcated donor forming weaker $\mathrm{C} 4-\mathrm{H} 4 A \cdots \mathrm{N} 2$ hydrogen bonds and $\mathrm{C} 4-\mathrm{H} 4 B \cdots \mathrm{Cg} 4$ interactions, Table 1 . In the chains, the mean plane of the oxadiazole ring is inclined at $10.7^{\circ}$ to $(101)$. The $\mathrm{N}-\mathrm{H} \cdots \mathrm{O}$ and $\mathrm{N}-\mathrm{H} \cdots \mathrm{S}$ hydrogen bonds also impose close $\mathrm{O} 1 \cdots \mathrm{N} 2(x, y-1, z)$ contacts of $2.9516(18) \AA$. Adjacent chains are further linked by $\mathrm{C} 3-\mathrm{H} 3 B \cdots \mathrm{S} 1$ hydrogen bonds that form inversion dimers, enclosing $R_{2}^{2}(12)$ rings. This combination of contacts stacks molecules along the $b$-axis direction, Fig. 3. Adjacent oxadiazole rings form dimers through $C g 1 \cdots C g 1^{\text {vi }} \pi-\pi$ contacts with centroid-to-centroid separations of 3.3931 (9) $\AA C g 1$ is the centroid of the $\mathrm{O} 1 / \mathrm{C} 2 /$ $\mathrm{N} 3 / \mathrm{N} 4 / \mathrm{C} 5$ ring; symmetry code: (vi) $1-x, 1-y, 1-z]$. These dimers are linked by much weaker $\mathrm{C} 12-\mathrm{H} 12 \cdots \mathrm{Cg} 4$ interactions, Table 1, forming chains along the ac diagonal, Fig. 4. This substantial array of contacts combines to form a threedimensional network structure, Fig. 5.

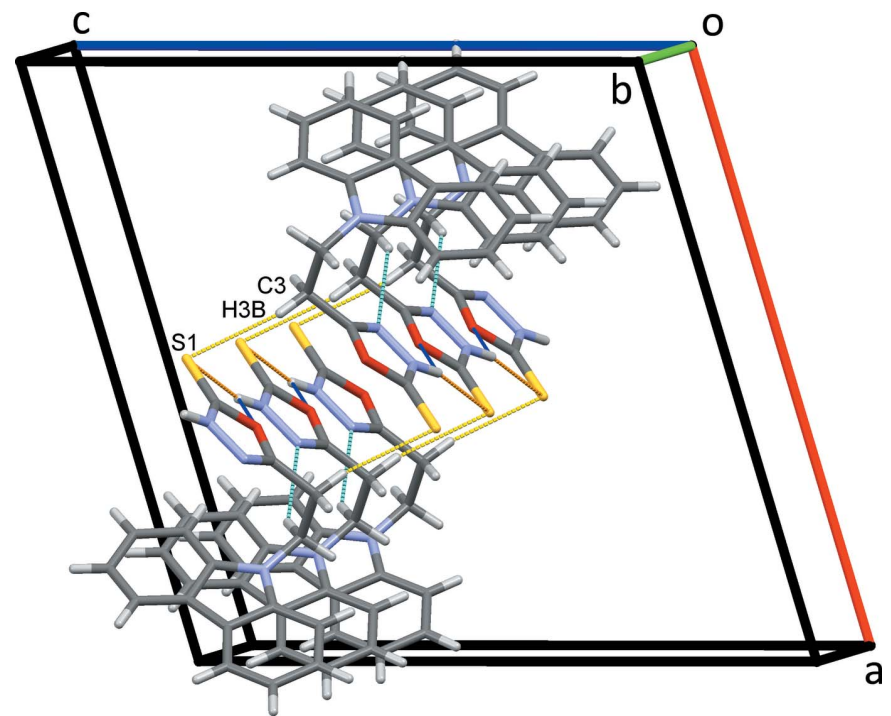

Figure 3

Inversion dimers formed by $\mathrm{C}-\mathrm{H} \cdots \mathrm{S}$ hydrogen bonds (dashed yellow lines) stacking rows of molecules of (I) along $b$. 


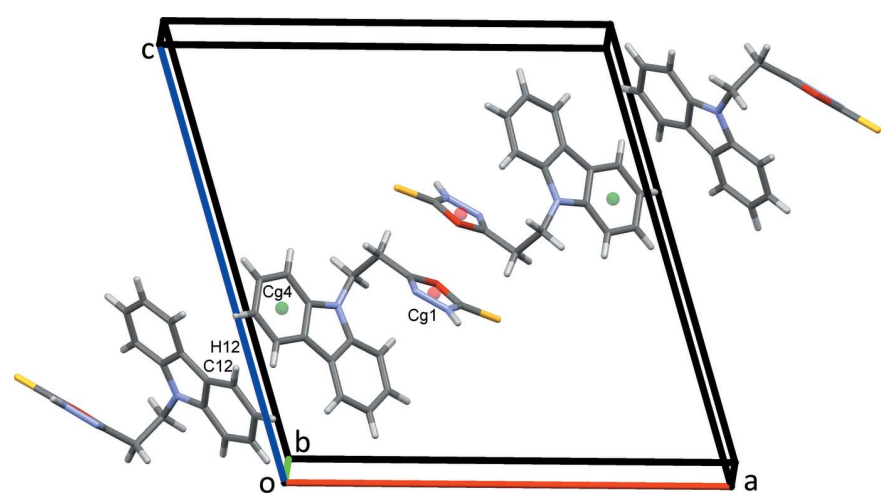

Figure 4

Chains of molecules of (I) along the ac diagonal. Centroid-centroid contacts are drawn as green dotted lines.

\section{Database survey}

Structures of carbazole derivatives abound in the Cambridge Structural Database (Version 5.38, November 2016 with one update; Groom et al., 2016) with 428 hits for solely organic molecules. Those with alkane chain substituents, at least two carbon atoms long on the pyrrole $\mathrm{N}$ atom, are less abundant with 47 hits for organic molecules alone. The simplest of these is $N$-ethyl carbazole itself (Kimura et al., 1985). This compound in fact appears in a number of manifestations as it seems to readily form co-crystals (Lee \& Wallwork, 1978; Hosomi et al., 2000; Matsuoka et al., 1988; Zhu et al., 2014). No examples were found of oxadiazole rings at the end of the alkane chains; indeed, the only derivatives with simple fivemembered rings in that position were dioxaborolane derivatives (Kalinin et al., 2003; Geier et al., 2009). In contrast, 1,3,4oxadiazole-2-thiones are far less abundant with only 29 unique organic structures reported. Furthermore, crystal structures of compounds with a chain of two or more methylene units bound to the 5-carbon are rare, with only three such structures found: 5-[2-(2-methoxyphenyl)ethyl]-1,3,4-oxadiazole-2(3H)thione and 5-[2-(4-methoxyphenyl)ethyl]-1,3,4-oxadiazole-

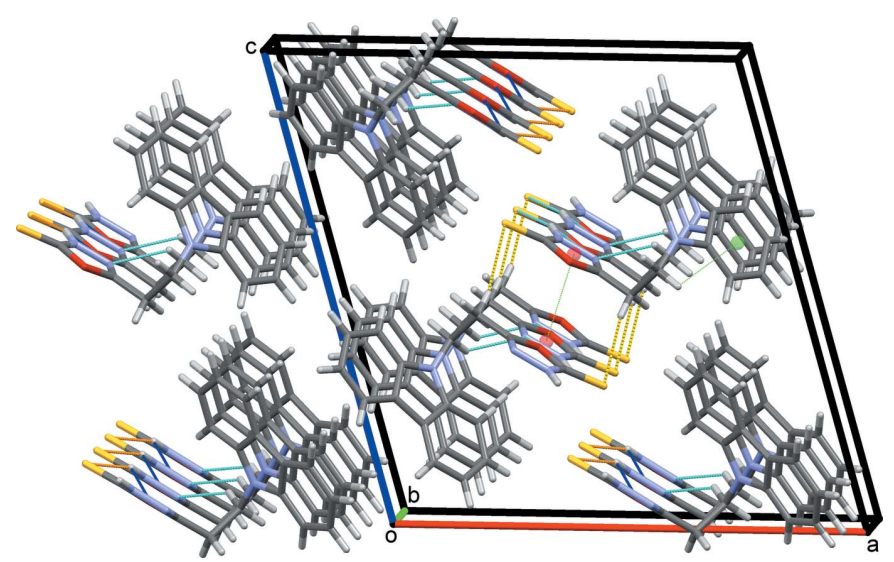

Figure 5

Overall packing of (I) viewed along the $b$-axis direction. Representative $\mathrm{C}-\mathrm{H} \cdots \pi$ hydrogen bonds and $\pi-\pi$ contacts are shown as green dotted lines.
Table 2

Experimental details.

\begin{tabular}{|c|c|}
\hline \multicolumn{2}{|l|}{ Crystal data } \\
\hline Chemical formula & $\mathrm{C}_{16} \mathrm{H}_{13} \mathrm{~N}_{3} \mathrm{OS}$ \\
\hline$M_{\mathrm{r}}$ & 295.35 \\
\hline Crystal system, space group & Monoclinic, $P 2_{1} / c$ \\
\hline Temperature $(\mathrm{K})$ & 100 \\
\hline$a, b, c(\AA)$ & $16.6868(5), 4.9600(1), 17.2353(6)$ \\
\hline$\beta\left(^{\circ}\right)$ & $105.909(3)$ \\
\hline$V\left(\AA^{3}\right)$ & $1371.87(7)$ \\
\hline$Z$ & 4 \\
\hline Radiation type & $\mathrm{Cu} K \alpha$ \\
\hline$\mu\left(\mathrm{mm}^{-1}\right)$ & 2.11 \\
\hline Crystal size $(\mathrm{mm})$ & $0.27 \times 0.15 \times 0.09$ \\
\hline \multicolumn{2}{|l|}{ Data collection } \\
\hline Diffractometer & $\begin{array}{l}\text { Agilent SuperNova, Dual, } \mathrm{Cu} \text { at } \\
\text { zero, Atlas }\end{array}$ \\
\hline Absorption correction & $\begin{array}{l}\text { Multi-scan (CrysAlis PRO; } \\
\quad \text { Agilent, 2014) }\end{array}$ \\
\hline$T_{\min }, T_{\max }$ & $0.763,1.000$ \\
\hline $\begin{array}{l}\text { No. of measured, independent and } \\
\text { observed }[I>2 \sigma(I)] \text { reflections }\end{array}$ & $11013,2849,2626$ \\
\hline$R_{\text {int }}$ & 0.063 \\
\hline$(\sin \theta / \lambda)_{\max }\left(\AA^{-1}\right)$ & 0.631 \\
\hline \multicolumn{2}{|l|}{ Refinement } \\
\hline$R\left[F^{2}>2 \sigma\left(F^{2}\right)\right], w R\left(F^{2}\right), S$ & $0.045,0.125,1.06$ \\
\hline No. of reflections & 2849 \\
\hline No. of parameters & 193 \\
\hline $\mathrm{H}$-atom treatment & $\begin{array}{l}\mathrm{H} \text { atoms treated by a mixture of } \\
\text { independent and constrained } \\
\text { refinement }\end{array}$ \\
\hline$\Delta \rho_{\max }, \Delta \rho_{\min }\left(\mathrm{e} \AA^{-3}\right)$ & $0.43,-0.48$ \\
\hline
\end{tabular}

Computer programs: CrysAlis PRO (Agilent, 2014), SHELXT (Sheldrick, 2015a), SHELXL2014 (Sheldrick, 2015b), TITAN2000 (Hunter \& Simpson, 1999), Mercury (Macrae et al., 2008), enCIFer (Allen et al., 2004), PLATON (Spek, 2009), publCIF (Westrip, 2010) and WinGX (Farrugia, 2012).

2(3H)-thione (Khan et al. 2014) and 5-[3-(quinolin-8-yloxy)propyl]-1,3,4-oxadiazole-2(3H)-thione (Zheng et al. 2006)

\section{Synthesis and crystallization}

A mixture of 3-(9H-carbazol-9-yl)propanehydrazide $(1.09 \mathrm{~g}$, $4 \mathrm{mmol})$ and carbon disulfide $(3 \mathrm{ml})$ in pyridine $(15 \mathrm{~mL})$ was heated under reflux on a water bath (333-343 K) overnight. The excess carbon disulfide was removed under reduced pressure and the reaction mixture was then poured into icecold water. The resulting precipitate was collected by filtration, washed with water, dried and recrystallized from mixed solvents of dioxane-water (1:1) to give (I) in $66 \%$ yield; m.p. 469-471 K. IR: NH, 3197, CH aromatic 3050, CH aliphatic $2940 \mathrm{~cm}^{-1} .{ }^{1} \mathrm{H}$ NMR: $\delta(\mathrm{ppm})\left(\mathrm{DMSO}-d_{6}\right) 2.35(t, 2 \mathrm{H}, \mathrm{CH} 2)$, $4.12(t, 2 \mathrm{H}, \mathrm{CH} 2), 7.35-8.38(m, 8 \mathrm{H}, \mathrm{Ar}-\mathrm{H}), 9.95(s, 1 \mathrm{H}, \mathrm{NH})$. ${ }^{13} \mathrm{C}$ NMR (100 MHz, DMSO- $d_{6}$, DEPT) $\delta$ (ppm): 34.9, 51.4, 109.6, 119.9, 121.4, 122.8, 156.8, 188.9. ms: $\mathrm{m} / z 295\left(M^{+}\right)$as molecular ion peak and base peak. Analysis calculated for $\mathrm{C}_{16}$ $\mathrm{H}_{13} \mathrm{~N}_{3} \mathrm{OS}$ (295.4): C, 65.06; H, 4.44; N, 5.42. Found: C, 65.38; H, $4.65 ; \mathrm{N}, 5.48$.

\section{Refinement}

Crystal data, data collection and structure refinement details are summarized in Table 2. The N-bound hydrogen atom was 
located in a difference-Fourier map and its coordinates refined with $U_{\text {iso }}=1.2 U_{\text {eq }}(\mathrm{N})$. All $\mathrm{H}$ atoms bound to $\mathrm{C}$ were refined using a riding model with $d(\mathrm{C}-\mathrm{H})=0.95 \AA$ and $U_{\text {iso }}(\mathrm{H})=$ $1.2 U_{\text {eq }}(\mathrm{C})$ for aromatic, $d(\mathrm{C}-\mathrm{H})=0.99 \AA$ and $U_{\text {iso }}(\mathrm{H})=$ $1.2 U_{\text {eq }}(\mathrm{C})$ for $\mathrm{CH}_{2} \mathrm{H}$ atoms.

\section{Acknowledgements}

We thank the University of Otago for purchase of the diffractometer and the Chemistry Department University of Otago for support of the work of JS.

\section{References}

Agilent (2014). CrysAlis PRO. Agilent Technologies, Yarnton, England.

Allen, F. H., Johnson, O., Shields, G. P., Smith, B. R. \& Towler, M. (2004). J. Appl. Cryst. 37, 335-338.

Billah, S. M. R., Christie, R. M. \& Shamey, R. (2008). Coloration Technol. 124, 223-228.

Farrugia, L. J. (2012). J. Appl. Cryst. 45, 849-854.

Fitilis, I., Fakis, M., Polyzos, I., Giannetas, V., Persephonis, P., Vellis, P. \& Mikroyannidis, J. (2007). Chem. Phys. Lett. 447, 300-304.

Geier, M. J., Vogels, C. M., Decken, A. \& Westcott, S. A. (2009). J. Organomet. Chem. 694, 3154-3159.

Ghoneim, O. M., Legere, J. A., Golbraikh, A., Tropsha, A. \& Booth, R. G. (2006). Bioorg. Med. Chem. 14, 6640-6658.
Groom, C. R., Bruno, I. J., Lightfoot, M. P. \& Ward, S. C. (2016). Acta Cryst. B72, 171-179.

Hosomi, H., Ohba, S. \& Ito, Y. (2000). Acta Cryst. C56, e147-e148.

Hunter, K. A. \& Simpson, J. (1999). TITAN2000. University of Otago, New Zealand.

Kalinin, A. V., Scherer, S. \& Snieckus, V. (2003). Angew. Chem. Int. Ed. 42, 3399-3404.

Khan, I., Ibrar, A. \& Simpson, J. (2014). CrystEngComm, 16, 164-174.

Kimura, T., Kai, Y., Yasuoka, N. \& Kasai, N. (1985). Bull. Chem. Soc. Jpn, 58, 2268-2271.

Lee, D. \& Wallwork, S. C. (1978). Acta Cryst. B34, 3604-3608.

Li, C., Liu, M., Pschirer, N. G., Baumgarten, M. \& Müllen, K. (2010). Chem. Rev. 110, 6817-6855.

Macrae, C. F., Bruno, I. J., Chisholm, J. A., Edgington, P. R., McCabe, P., Pidcock, E., Rodriguez-Monge, L., Taylor, R., van de Streek, J. \& Wood, P. A. (2008). J. Appl. Cryst. 41, 466-470.

Matsuoka, M., Han, L., Kitao, T., Mochizuki, S. \& Nakatsu, K. (1988). Chem. Lett. 17, 905-908.

Peng, Q., Liu, X., Qin, Y., Xu, J., Li, M. \& Dai, L. (2011). J. Mater. Chem. 21, 7714-7722.

Sheldrick, G. M. (2015a). Acta Cryst. A71, 3-8.

Sheldrick, G. M. (2015b). Acta Cryst. C71, 3-8.

Spek, A. L. (2009). Acta Cryst. D65, 148-155.

TePaske, M. R., Gloer, J. B., Wicklow, D. T. \& Dowd, P. F. (1989). Tetrahedron Lett. 30, 5965-5968.

Westrip, S. P. (2010). J. Appl. Cryst. 43, 920-925.

Zheng, Z.-B., Wu, R.-T., Lu, J.-R. \& Sun, Y.-F. (2006). Acta Cryst. E62, o4293-o4295.

Zhu, Q., Gao, Y. J., Gao, H. Y. \& Jin, W. J. (2014). J. Photochem. Photobiol. Chem. 289, 31-38. 


\section{supporting information}

Acta Cryst. (2017). E73, 1066-1069 [https://doi.org/10.1107/S2056989017009252]

Crystal structure of 5-[2-(9H-carbazol-9-yl)ethyl]-1,3,4-oxadiazole-2(3H)-thione

Jim Simpson, Shaaban K. Mohamed, Talaat I. El-Emary and Mustafa R. Albayati

Computing details

Data collection: CrysAlis PRO (Agilent, 2014); cell refinement: CrysAlis PRO (Agilent, 2014); data reduction: CrysAlis $P R O$ (Agilent, 2014); program(s) used to solve structure: SHELXT (Sheldrick, 2015a); program(s) used to refine structure: SHELXL2014 (Sheldrick, 2015b) and TITAN2000 (Hunter \& Simpson, 1999); molecular graphics: Mercury (Macrae et al., 2008); software used to prepare material for publication: SHELXL2014 (Sheldrick, 2015b), enCIFer (Allen et al., 2004), PLATON (Spek, 2009), publCIF (Westrip, 2010) and WinGX (Farrugia, 2012).

5-[2-(9H-carbazol-9-yl)ethyl]-1,3,4-oxadiazole-2(3H)-thione

Crystal data

$\mathrm{C}_{16} \mathrm{H}_{13} \mathrm{~N}_{3} \mathrm{OS}$

$M_{r}=295.35$

Monoclinic, $P 2{ }_{1} / c$

$a=16.6868(5) \AA$

$b=4.9600(1) \AA$

$c=17.2353(6) \AA$

$\beta=105.909(3)^{\circ}$

$V=1371.87(7) \AA^{3}$

$Z=4$

\section{Data collection}

Agilent SuperNova, Dual, $\mathrm{Cu}$ at zero, Atlas diffractometer

Radiation source: SuperNova $(\mathrm{Cu}) \mathrm{X}$-ray Source

Detector resolution: 5.1725 pixels $\mathrm{mm}^{-1}$ $\omega$ scans

Absorption correction: multi-scan

(CrysAlis PRO; Agilent, 2014)

$T_{\min }=0.763, T_{\max }=1.000$

Refinement

Refinement on $F^{2}$

Least-squares matrix: full

$R\left[F^{2}>2 \sigma\left(F^{2}\right)\right]=0.045$

$w R\left(F^{2}\right)=0.125$

$S=1.06$

2849 reflections

193 parameters

0 restraints
$F(000)=616$

$D_{\mathrm{x}}=1.430 \mathrm{Mg} \mathrm{m}^{-3}$

$\mathrm{Cu} K \alpha$ radiation, $\lambda=1.54184 \AA$

Cell parameters from 7352 reflections

$\theta=6.5-76.5^{\circ}$

$\mu=2.11 \mathrm{~mm}^{-1}$

$T=100 \mathrm{~K}$

Plate, colourless

$0.27 \times 0.15 \times 0.09 \mathrm{~mm}$

11013 measured reflections

2849 independent reflections

2626 reflections with $I>2 \sigma(I)$

$R_{\text {int }}=0.063$

$\theta_{\text {max }}=76.5^{\circ}, \theta_{\min }=5.3^{\circ}$

$h=-20 \rightarrow 20$

$k=-4 \rightarrow 6$

$l=-20 \rightarrow 21$

Hydrogen site location: mixed

$\mathrm{H}$ atoms treated by a mixture of independent and constrained refinement

$w=1 /\left[\sigma^{2}\left(F_{\mathrm{o}}^{2}\right)+(0.079 P)^{2}+0.5548 P\right]$

where $P=\left(F_{\mathrm{o}}{ }^{2}+2 F_{\mathrm{c}}{ }^{2}\right) / 3$

$(\Delta / \sigma)_{\max }=0.001$

$\Delta \rho_{\max }=0.43$ e $\AA^{-3}$

$\Delta \rho_{\min }=-0.48 \mathrm{e} \AA^{-3}$ 


\section{Special details}

Geometry. All esds (except the esd in the dihedral angle between two 1.s. planes) are estimated using the full covariance matrix. The cell esds are taken into account individually in the estimation of esds in distances, angles and torsion angles; correlations between esds in cell parameters are only used when they are defined by crystal symmetry. An approximate (isotropic) treatment of cell esds is used for estimating esds involving l.s. planes.

Fractional atomic coordinates and isotropic or equivalent isotropic displacement parameters $\left(\AA^{2}\right)$

\begin{tabular}{|c|c|c|c|c|}
\hline & $x$ & $y$ & $z$ & $U_{\text {iso }} * / U_{\text {eq }}$ \\
\hline $\mathrm{O} 1$ & $0.55153(7)$ & $0.1963(2)$ & $0.58574(7)$ & 0.0155 (3) \\
\hline $\mathrm{C} 1$ & $0.50674(9)$ & $0.3454(3)$ & $0.62638(9)$ & $0.0151(3)$ \\
\hline $\mathrm{S} 1$ & $0.43636(2)$ & $0.21061(9)$ & $0.66526(2)$ & $0.01955(16)$ \\
\hline N1 & $0.53275(8)$ & $0.5979(3)$ & $0.62294(8)$ & $0.0158(3)$ \\
\hline $\mathrm{H} 1 \mathrm{~N}$ & $0.5152(14)$ & $0.740(5)$ & $0.6449(14)$ & $0.019 *$ \\
\hline N2 & $0.59279(8)$ & $0.6190(3)$ & $0.58088(8)$ & $0.0168(3)$ \\
\hline $\mathrm{C} 2$ & $0.60197(9)$ & $0.3745(3)$ & $0.56023(9)$ & $0.0146(3)$ \\
\hline $\mathrm{C} 3$ & $0.66006(10)$ & $0.2674(3)$ & $0.51646(10)$ & 0.0175 (3) \\
\hline H6A & 0.6797 & 0.4173 & 0.4885 & $0.021 *$ \\
\hline H3B & 0.6301 & 0.1372 & 0.4751 & $0.021 *$ \\
\hline $\mathrm{C} 4$ & $0.73573(9)$ & $0.1269(3)$ & $0.57370(10)$ & $0.0168(3)$ \\
\hline $\mathrm{H} 4 \mathrm{~A}$ & 0.7160 & -0.0214 & 0.6022 & $0.020 *$ \\
\hline H4B & 0.7707 & 0.0470 & 0.5416 & $0.020 *$ \\
\hline N3 & $0.78545(8)$ & 0.3109 (3) & $0.63217(8)$ & 0.0151 (3) \\
\hline C5 & $0.77515(9)$ & $0.3625(3)$ & $0.70793(9)$ & $0.0154(3)$ \\
\hline $\mathrm{C} 6$ & $0.72386(10)$ & $0.2314(4)$ & $0.74787(10)$ & $0.0201(4)$ \\
\hline H6 & 0.6892 & 0.0851 & 0.7237 & $0.024 *$ \\
\hline $\mathrm{C} 7$ & $0.72578(11)$ & $0.3239(4)$ & $0.82431(11)$ & $0.0273(4)$ \\
\hline H7 & 0.6917 & 0.2390 & 0.8531 & $0.033^{*}$ \\
\hline $\mathrm{C} 8$ & $0.77677(13)$ & $0.5393(4)$ & $0.85996(10)$ & 0.0309 (4) \\
\hline H8 & 0.7763 & 0.5987 & 0.9122 & $0.037 *$ \\
\hline C9 & 0.82767 (11) & 0.6669 (4) & $0.82050(11)$ & $0.0262(4)$ \\
\hline H9 & 0.8621 & 0.8130 & 0.8452 & $0.031 *$ \\
\hline $\mathrm{C} 10$ & $0.82777(9)$ & $0.5777(3)$ & $0.74355(10)$ & $0.0182(3)$ \\
\hline $\mathrm{C} 11$ & 0.87157 (9) & 0.6589 (3) & $0.68573(10)$ & $0.0190(3)$ \\
\hline $\mathrm{C} 12$ & $0.93139(10)$ & $0.8561(4)$ & $0.68605(12)$ & $0.0274(4)$ \\
\hline H12 & 0.9509 & 0.9717 & 0.7312 & $0.033^{*}$ \\
\hline $\mathrm{C} 13$ & $0.96158(11)$ & 0.8797 (4) & $0.61920(13)$ & $0.0327(5)$ \\
\hline H13 & 1.0023 & 1.0130 & 0.6188 & $0.039 *$ \\
\hline $\mathrm{C} 14$ & $0.93329(11)$ & $0.7113(4)$ & $0.55235(13)$ & $0.0296(4)$ \\
\hline H14 & 0.9556 & 0.7320 & 0.5076 & $0.036^{*}$ \\
\hline $\mathrm{C} 15$ & $0.87311(10)$ & $0.5133(4)$ & 0.54963 (11) & $0.0223(4)$ \\
\hline H15 & 0.8535 & 0.3998 & 0.5040 & $0.027 *$ \\
\hline C16 & $0.84324(9)$ & $0.4908(3)$ & $0.61765(10)$ & $0.0167(3)$ \\
\hline
\end{tabular}

Atomic displacement parameters $\left(\AA^{2}\right)$

\begin{tabular}{lllllll}
\hline & $U^{11}$ & $U^{22}$ & $U^{33}$ & $U^{12}$ & $U^{13}$ & $U^{23}$ \\
\hline $\mathrm{O} 1$ & $0.0145(5)$ & $0.0103(5)$ & $0.0207(6)$ & $-0.0003(4)$ & $0.0030(4)$ & $-0.0018(4)$
\end{tabular}




$\begin{array}{lllllll}\text { C1 } & 0.0127(7) & 0.0132(7) & 0.0162(7) & 0.0011(6) & -0.0014(5) & -0.0011(5) \\ \text { S1 } & 0.0185(2) & 0.0185(3) & 0.0215(2) & -0.00502(14) & 0.00544(16) & -0.00070(14) \\ \text { N1 } & 0.0149(6) & 0.0108(7) & 0.0222(6) & -0.0002(5) & 0.0057(5) & -0.0018(5) \\ \text { N2 } & 0.0139(6) & 0.0136(7) & 0.0230(7) & -0.0003(5) & 0.0052(5) & 0.0000(5) \\ \text { C2 } & 0.0116(6) & 0.0129(7) & 0.0166(7) & -0.0004(6) & -0.0006(5) & 0.0005(6) \\ \text { C3 } & 0.0155(7) & 0.0185(8) & 0.0173(7) & 0.0004(6) & 0.0023(6) & -0.0030(6) \\ \text { C4 } & 0.0136(7) & 0.0144(7) & 0.0217(7) & 0.0006(6) & 0.0035(6) & -0.0029(6) \\ \text { N3 } & 0.0110(6) & 0.0156(7) & 0.0184(7) & -0.0021(5) & 0.0035(5) & -0.0013(5) \\ \text { C5 } & 0.0114(6) & 0.0162(8) & 0.0167(7) & 0.0050(6) & 0.0006(5) & 0.0015(6) \\ \text { C6 } & 0.0144(7) & 0.0226(8) & 0.0231(8) & 0.0053(6) & 0.0047(6) & 0.0050(6) \\ \text { C7 } & 0.0261(9) & 0.0351(11) & 0.0225(9) & 0.0143(8) & 0.0097(7) & 0.0099(7) \\ \text { C8 } & 0.0371(10) & 0.0367(11) & 0.0166(8) & 0.0175(8) & 0.0035(7) & 0.0002(7) \\ \text { C9 } & 0.0254(8) & 0.0242(9) & 0.0215(8) & 0.0089(7) & -0.0061(6) & -0.0032(7) \\ \text { C10 } & 0.0131(7) & 0.0175(8) & 0.0188(7) & 0.0048(6) & -0.0047(5) & 0.0011(6) \\ \text { C11 } & 0.0098(6) & 0.0157(8) & 0.0259(8) & 0.0017(6) & -0.0048(6) & 0.0030(6) \\ \text { C12 } & 0.0145(7) & 0.0189(9) & 0.0395(10) & -0.0035(7) & -0.0081(7) & 0.0056(8) \\ \text { C13 } & 0.0122(7) & 0.0283(10) & 0.0525(12) & -0.0042(7) & -0.0001(7) & 0.0169(9) \\ \text { C14 } & 0.0167(8) & 0.0314(11) & 0.0424(11) & 0.0031(7) & 0.0107(7) & 0.0164(8) \\ \text { C15 } & 0.0173(7) & 0.0217(9) & 0.0295(8) & 0.0040(6) & 0.0090(6) & 0.0063(7) \\ \text { C16 } & 0.0089(6) & 0.0152(8) & 0.0247(8) & 0.0025(5) & 0.0024(5) & 0.0048(6)\end{array}$

Geometric parameters $\left(\AA,{ }^{\circ}\right)$

\begin{tabular}{|c|c|c|c|}
\hline $\mathrm{O} 1-\mathrm{C} 1$ & $1.3723(18)$ & $\mathrm{C} 6-\mathrm{C} 7$ & $1.387(3)$ \\
\hline $\mathrm{O} 1-\mathrm{C} 2$ & $1.3732(18)$ & C6- 6 6 & 0.9500 \\
\hline $\mathrm{C} 1-\mathrm{N} 1$ & $1.332(2)$ & $\mathrm{C} 7-\mathrm{C} 8$ & $1.399(3)$ \\
\hline $\mathrm{C} 1-\mathrm{S} 1$ & $1.6452(16)$ & $\mathrm{C} 7-\mathrm{H} 7$ & 0.9500 \\
\hline $\mathrm{N} 1-\mathrm{N} 2$ & $1.3922(18)$ & $\mathrm{C} 8-\mathrm{C} 9$ & $1.379(3)$ \\
\hline $\mathrm{N} 1-\mathrm{H} 1 \mathrm{~N}$ & $0.89(2)$ & $\mathrm{C} 8-\mathrm{H} 8$ & 0.9500 \\
\hline $\mathrm{N} 2-\mathrm{C} 2$ & $1.285(2)$ & $\mathrm{C} 9-\mathrm{C} 10$ & $1.398(2)$ \\
\hline $\mathrm{N} 2-\mathrm{O} 1^{\mathrm{i}}$ & $2.9516(18)$ & C9- & 0.9500 \\
\hline $\mathrm{C} 2-\mathrm{C} 3$ & $1.480(2)$ & $\mathrm{C} 10-\mathrm{C} 11$ & $1.445(2)$ \\
\hline $\mathrm{C} 3-\mathrm{C} 4$ & $1.540(2)$ & $\mathrm{C} 11-\mathrm{C} 12$ & $1.396(2)$ \\
\hline $\mathrm{C} 3-\mathrm{H} 6 \mathrm{~A}$ & 0.9900 & $\mathrm{C} 11-\mathrm{C} 16$ & $1.411(2)$ \\
\hline $\mathrm{C} 3-\mathrm{H} 3 \mathrm{~B}$ & 0.9900 & $\mathrm{C} 12-\mathrm{C} 13$ & $1.383(3)$ \\
\hline $\mathrm{C} 4-\mathrm{N} 3$ & $1.442(2)$ & $\mathrm{C} 12-\mathrm{H} 12$ & 0.9500 \\
\hline $\mathrm{C} 4-\mathrm{H} 4 \mathrm{~A}$ & 0.9900 & $\mathrm{C} 13-\mathrm{C} 14$ & $1.396(3)$ \\
\hline $\mathrm{C} 4-\mathrm{H} 4 \mathrm{~B}$ & 0.9900 & $\mathrm{C} 13-\mathrm{H} 13$ & 0.9500 \\
\hline $\mathrm{N} 3-\mathrm{C} 16$ & $1.386(2)$ & $\mathrm{C} 14-\mathrm{C} 15$ & $1.396(3)$ \\
\hline $\mathrm{N} 3-\mathrm{C} 5$ & $1.387(2)$ & $\mathrm{C} 14-\mathrm{H} 14$ & 0.9500 \\
\hline $\mathrm{C} 5-\mathrm{C} 6$ & $1.397(2)$ & $\mathrm{C} 15-\mathrm{C} 16$ & $1.398(2)$ \\
\hline $\mathrm{C} 5-\mathrm{C} 10$ & $1.411(2)$ & $\mathrm{C} 15-\mathrm{H} 15$ & 0.9500 \\
\hline $\mathrm{C} 1-\mathrm{O} 1-\mathrm{C} 2$ & $106.49(12)$ & $\mathrm{C} 7-\mathrm{C} 6-\mathrm{H} 6$ & 121.4 \\
\hline $\mathrm{N} 1-\mathrm{C} 1-\mathrm{O} 1$ & $104.73(13)$ & $\mathrm{C} 5-\mathrm{C} 6-\mathrm{H} 6$ & 121.4 \\
\hline $\mathrm{N} 1-\mathrm{C} 1-\mathrm{S} 1$ & $132.68(13)$ & $\mathrm{C} 6-\mathrm{C} 7-\mathrm{C} 8$ & $121.53(17)$ \\
\hline $\mathrm{O} 1-\mathrm{C} 1-\mathrm{S} 1$ & $122.56(12)$ & $\mathrm{C} 6-\mathrm{C} 7-\mathrm{H} 7$ & 119.2 \\
\hline $\mathrm{C} 1-\mathrm{N} 1-\mathrm{N} 2$ & $112.56(13)$ & $\mathrm{C} 8-\mathrm{C} 7-\mathrm{H} 7$ & 119.2 \\
\hline
\end{tabular}




\begin{tabular}{|c|c|c|c|}
\hline $\mathrm{C} 1-\mathrm{N} 1-\mathrm{H} 1 \mathrm{~N}$ & $125.4(14)$ & $\mathrm{C} 9-\mathrm{C} 8-\mathrm{C} 7$ & $121.11(17)$ \\
\hline $\mathrm{N} 2-\mathrm{N} 1-\mathrm{H} 1 \mathrm{~N}$ & $122.1(14)$ & $\mathrm{C} 9-\mathrm{C} 8-\mathrm{H} 8$ & 119.4 \\
\hline $\mathrm{C} 2-\mathrm{N} 2-\mathrm{N} 1$ & $103.34(13)$ & $\mathrm{C} 7-\mathrm{C} 8-\mathrm{H} 8$ & 119.4 \\
\hline $\mathrm{C} 2-\mathrm{N} 2-\mathrm{O} 1^{\mathrm{i}}$ & $165.93(11)$ & $\mathrm{C} 8-\mathrm{C} 9-\mathrm{C} 10$ & $118.93(18)$ \\
\hline $\mathrm{N} 1-\mathrm{N} 2-\mathrm{O} 1^{\mathrm{i}}$ & $81.45(9)$ & $\mathrm{C} 8-\mathrm{C} 9-\mathrm{H} 9$ & 120.5 \\
\hline $\mathrm{N} 2-\mathrm{C} 2-\mathrm{O} 1$ & $112.87(13)$ & $\mathrm{C} 10-\mathrm{C} 9-\mathrm{H} 9$ & 120.5 \\
\hline $\mathrm{N} 2-\mathrm{C} 2-\mathrm{C} 3$ & $128.62(15)$ & $\mathrm{C} 9-\mathrm{C} 10-\mathrm{C} 5$ & $119.30(16)$ \\
\hline $\mathrm{O} 1-\mathrm{C} 2-\mathrm{C} 3$ & $118.48(14)$ & $\mathrm{C} 9-\mathrm{C} 10-\mathrm{C} 11$ & $134.28(17)$ \\
\hline $\mathrm{C} 2-\mathrm{C} 3-\mathrm{C} 4$ & $111.86(13)$ & $\mathrm{C} 5-\mathrm{C} 10-\mathrm{C} 11$ & $106.41(14)$ \\
\hline $\mathrm{C} 2-\mathrm{C} 3-\mathrm{H} 6 \mathrm{~A}$ & 109.2 & $\mathrm{C} 12-\mathrm{C} 11-\mathrm{C} 16$ & $119.57(17)$ \\
\hline $\mathrm{C} 4-\mathrm{C} 3-\mathrm{H} 6 \mathrm{~A}$ & 109.2 & $\mathrm{C} 12-\mathrm{C} 11-\mathrm{C} 10$ & $133.50(17)$ \\
\hline $\mathrm{C} 2-\mathrm{C} 3-\mathrm{H} 3 \mathrm{~B}$ & 109.2 & $\mathrm{C} 16-\mathrm{C} 11-\mathrm{C} 10$ & $106.93(14)$ \\
\hline $\mathrm{C} 4-\mathrm{C} 3-\mathrm{H} 3 \mathrm{~B}$ & 109.2 & $\mathrm{C} 13-\mathrm{C} 12-\mathrm{C} 11$ & $118.63(19)$ \\
\hline $\mathrm{H} 6 \mathrm{~A}-\mathrm{C} 3-\mathrm{H} 3 \mathrm{~B}$ & 107.9 & $\mathrm{C} 13-\mathrm{C} 12-\mathrm{H} 12$ & 120.7 \\
\hline $\mathrm{N} 3-\mathrm{C} 4-\mathrm{C} 3$ & $112.02(13)$ & $\mathrm{C} 11-\mathrm{C} 12-\mathrm{H} 12$ & 120.7 \\
\hline $\mathrm{N} 3-\mathrm{C} 4-\mathrm{H} 4 \mathrm{~A}$ & 109.2 & $\mathrm{C} 12-\mathrm{C} 13-\mathrm{C} 14$ & $121.24(17)$ \\
\hline $\mathrm{C} 3-\mathrm{C} 4-\mathrm{H} 4 \mathrm{~A}$ & 109.2 & $\mathrm{C} 12-\mathrm{C} 13-\mathrm{H} 13$ & 119.4 \\
\hline $\mathrm{N} 3-\mathrm{C} 4-\mathrm{H} 4 \mathrm{~B}$ & 109.2 & $\mathrm{C} 14-\mathrm{C} 13-\mathrm{H} 13$ & 119.4 \\
\hline $\mathrm{C} 3-\mathrm{C} 4-\mathrm{H} 4 \mathrm{~B}$ & 109.2 & $\mathrm{C} 15-\mathrm{C} 14-\mathrm{C} 13$ & $121.73(18)$ \\
\hline $\mathrm{H} 4 \mathrm{~A}-\mathrm{C} 4-\mathrm{H} 4 \mathrm{~B}$ & 107.9 & $\mathrm{C} 15-\mathrm{C} 14-\mathrm{H} 14$ & 119.1 \\
\hline $\mathrm{C} 16-\mathrm{N} 3-\mathrm{C} 5$ & $108.85(13)$ & $\mathrm{C} 13-\mathrm{C} 14-\mathrm{H} 14$ & 119.1 \\
\hline $\mathrm{C} 16-\mathrm{N} 3-\mathrm{C} 4$ & $125.23(14)$ & $\mathrm{C} 14-\mathrm{C} 15-\mathrm{C} 16$ & $116.51(17)$ \\
\hline $\mathrm{C} 5-\mathrm{N} 3-\mathrm{C} 4$ & $125.38(13)$ & $\mathrm{C} 14-\mathrm{C} 15-\mathrm{H} 15$ & 121.7 \\
\hline $\mathrm{N} 3-\mathrm{C} 5-\mathrm{C} 6$ & $128.95(15)$ & $\mathrm{C} 16-\mathrm{C} 15-\mathrm{H} 15$ & 121.7 \\
\hline $\mathrm{N} 3-\mathrm{C} 5-\mathrm{C} 10$ & $109.05(14)$ & $\mathrm{N} 3-\mathrm{C} 16-\mathrm{C} 15$ & $128.93(16)$ \\
\hline $\mathrm{C} 6-\mathrm{C} 5-\mathrm{C} 10$ & $122.00(15)$ & $\mathrm{N} 3-\mathrm{C} 16-\mathrm{C} 11$ & $108.74(14)$ \\
\hline $\mathrm{C} 7-\mathrm{C} 6-\mathrm{C} 5$ & $117.12(17)$ & $\mathrm{C} 15-\mathrm{C} 16-\mathrm{C} 11$ & $122.32(16)$ \\
\hline $\mathrm{C} 2-\mathrm{O} 1-\mathrm{C} 1-\mathrm{N} 1$ & $-0.16(15)$ & $\mathrm{C} 8-\mathrm{C} 9-\mathrm{C} 10-\mathrm{C} 5$ & $0.8(2)$ \\
\hline $\mathrm{C} 2-\mathrm{O} 1-\mathrm{C} 1-\mathrm{S} 1$ & $178.36(11)$ & $\mathrm{C} 8-\mathrm{C} 9-\mathrm{C} 10-\mathrm{C} 11$ & $-179.84(17)$ \\
\hline $\mathrm{O} 1-\mathrm{C} 1-\mathrm{N} 1-\mathrm{N} 2$ & $0.37(16)$ & $\mathrm{N} 3-\mathrm{C} 5-\mathrm{C} 10-\mathrm{C} 9$ & $179.65(14)$ \\
\hline $\mathrm{S} 1-\mathrm{C} 1-\mathrm{N} 1-\mathrm{N} 2$ & $-177.93(12)$ & $\mathrm{C} 6-\mathrm{C} 5-\mathrm{C} 10-\mathrm{C} 9$ & $-1.3(2)$ \\
\hline $\mathrm{C} 1-\mathrm{N} 1-\mathrm{N} 2-\mathrm{C} 2$ & $-0.44(17)$ & $\mathrm{N} 3-\mathrm{C} 5-\mathrm{C} 10-\mathrm{C} 11$ & $0.15(17)$ \\
\hline $\mathrm{C} 1-\mathrm{N} 1-\mathrm{N} 2-\mathrm{O} 1^{\mathrm{i}}$ & $166.09(12)$ & $\mathrm{C} 6-\mathrm{C} 5-\mathrm{C} 10-\mathrm{C} 11$ & $179.21(14)$ \\
\hline $\mathrm{N} 1-\mathrm{N} 2-\mathrm{C} 2-\mathrm{O} 1$ & $0.32(17)$ & $\mathrm{C} 9-\mathrm{C} 10-\mathrm{C} 11-\mathrm{C} 12$ & $1.0(3)$ \\
\hline $\mathrm{O} 1-\mathrm{i} 2-\mathrm{C} 2-\mathrm{O} 1$ & $-108.2(4)$ & $\mathrm{C} 5-\mathrm{C} 10-\mathrm{C} 11-\mathrm{C} 12$ & $-179.56(18)$ \\
\hline $\mathrm{N} 1-\mathrm{N} 2-\mathrm{C} 2-\mathrm{C} 3$ & $-177.54(14)$ & $\mathrm{C} 9-\mathrm{C} 10-\mathrm{C} 11-\mathrm{C} 16$ & $-179.05(17)$ \\
\hline $\mathrm{O} 1-\mathrm{i} 2-\mathrm{C} 2-\mathrm{C} 3$ & $73.9(5)$ & $\mathrm{C} 5-\mathrm{C} 10-\mathrm{C} 11-\mathrm{C} 16$ & $0.35(17)$ \\
\hline $\mathrm{C} 1-\mathrm{O} 1-\mathrm{C} 2-\mathrm{N} 2$ & $-0.11(17)$ & $\mathrm{C} 16-\mathrm{C} 11-\mathrm{C} 12-\mathrm{C} 13$ & $-0.3(2)$ \\
\hline $\mathrm{C} 1-\mathrm{O} 1-\mathrm{C} 2-\mathrm{C} 3$ & $177.99(13)$ & $\mathrm{C} 10-\mathrm{C} 11-\mathrm{C} 12-\mathrm{C} 13$ & $179.55(17)$ \\
\hline $\mathrm{N} 2-\mathrm{C} 2-\mathrm{C} 3-\mathrm{C} 4$ & $102.47(19)$ & $\mathrm{C} 11-\mathrm{C} 12-\mathrm{C} 13-\mathrm{C} 14$ & $0.1(3)$ \\
\hline $\mathrm{O} 1-\mathrm{C} 2-\mathrm{C} 3-\mathrm{C} 4$ & $-75.29(17)$ & $\mathrm{C} 12-\mathrm{C} 13-\mathrm{C} 14-\mathrm{C} 15$ & $0.4(3)$ \\
\hline $\mathrm{C} 2-\mathrm{C} 3-\mathrm{C} 4-\mathrm{N} 3$ & $-62.96(18)$ & $\mathrm{C} 13-\mathrm{C} 14-\mathrm{C} 15-\mathrm{C} 16$ & $-0.6(3)$ \\
\hline $\mathrm{C} 3-\mathrm{C} 4-\mathrm{N} 3-\mathrm{C} 16$ & $-79.78(18)$ & $\mathrm{C} 5-\mathrm{N} 3-\mathrm{C} 16-\mathrm{C} 15$ & $179.80(15)$ \\
\hline $\mathrm{C} 3-\mathrm{C} 4-\mathrm{N} 3-\mathrm{C} 5$ & $90.85(18)$ & $\mathrm{C} 4-\mathrm{N} 3-\mathrm{C} 16-\mathrm{C} 15$ & $-8.3(3)$ \\
\hline $\mathrm{C} 16-\mathrm{N} 3-\mathrm{C} 5-\mathrm{C} 6$ & $-179.58(15)$ & $\mathrm{C} 5-\mathrm{N} 3-\mathrm{C} 16-\mathrm{C} 11$ & $0.82(17)$ \\
\hline $\mathrm{C} 4-\mathrm{N} 3-\mathrm{C} 5-\mathrm{C} 6$ & $8.5(3)$ & $\mathrm{C} 4-\mathrm{N} 3-\mathrm{C} 16-\mathrm{C} 11$ & $172.75(14)$ \\
\hline $\mathrm{C} 16-\mathrm{N} 3-\mathrm{C} 5-\mathrm{C} 10$ & $-0.60(17)$ & $\mathrm{C} 14-\mathrm{C} 15-\mathrm{C} 16-\mathrm{N} 3$ & $-178.53(16)$ \\
\hline
\end{tabular}




$\begin{array}{llll}\mathrm{C} 4-\mathrm{N} 3-\mathrm{C} 5-\mathrm{C} 10 & -172.52(14) & \mathrm{C} 14-\mathrm{C} 15-\mathrm{C} 16-\mathrm{C} 11 & 0.3(2) \\ \mathrm{N} 3-\mathrm{C} 5-\mathrm{C} 6-\mathrm{C} 7 & 179.70(15) & \mathrm{C} 12-\mathrm{C} 11-\mathrm{C} 16-\mathrm{N} 3 & 179.20(14) \\ \mathrm{C} 10-\mathrm{C} 5-\mathrm{C} 6-\mathrm{C} 7 & 0.8(2) & \mathrm{C} 10-\mathrm{C} 11-\mathrm{C} 16-\mathrm{N} 3 & -0.72(17) \\ \mathrm{C} 5-\mathrm{C} 6-\mathrm{C} 7-\mathrm{C} 8 & 0.0(2) & \mathrm{C} 12-\mathrm{C} 11-\mathrm{C} 16-\mathrm{C} 15 & 0.1(2) \\ \mathrm{C} 6-\mathrm{C} 7-\mathrm{C} 8-\mathrm{C} 9 & -0.5(3) & \mathrm{C} 10-\mathrm{C} 11-\mathrm{C} 16-\mathrm{C} 15 & -179.78(14) \\ \mathrm{C} 7-\mathrm{C} 8-\mathrm{C} 9-\mathrm{C} 10 & 0.0(3) & & \end{array}$

Symmetry code: (i) $x, y+1, z$.

Hydrogen-bond geometry $\left(\AA,{ }^{\circ}\right)$

$\mathrm{Cg} 4$ is the centroid of the $\mathrm{C} 11-\mathrm{C} 16$ ring.

\begin{tabular}{lllll}
\hline$D-\mathrm{H} \cdots A$ & $D-\mathrm{H}$ & $\mathrm{H} \cdots A$ & $D \cdots A$ & $D-\mathrm{H} \cdots A$ \\
\hline $\mathrm{N} 1-\mathrm{H} 1 N \cdots \mathrm{S} 1^{\mathrm{i}}$ & $0.89(2)$ & $2.75(2)$ & $3.6053(14)$ & $162.8(19)$ \\
$\mathrm{N} 1-\mathrm{H} 1 N \cdots \mathrm{O} 1^{\mathrm{i}}$ & $0.89(2)$ & $2.62(2)$ & $3.0707(18)$ & $112.5(16)$ \\
$\mathrm{C} 3-\mathrm{H} 3 B \cdots \mathrm{S} 1^{i i}$ & 0.99 & 2.93 & $3.9061(16)$ & 169 \\
$\mathrm{C} 4-\mathrm{H} 4 A \cdots \mathrm{N} 2^{\mathrm{iii}}$ & 0.99 & 2.67 & $3.495(2)$ & 141 \\
$\mathrm{C} 4-\mathrm{H} 4 B \cdots C g 4^{\mathrm{iii}}$ & 0.99 & 2.87 & $3.4577(17)$ & 119 \\
$\mathrm{C} 12-\mathrm{H} 12 \cdots C g 4^{\mathrm{i}}$ & 0.95 & 3.22 & $4.073(2)$ & 151
\end{tabular}

Symmetry codes: (i) $x, y+1, z$; (ii) $-x+1,-y,-z+1$; (iii) $x, y-1, z$. 